\title{
UNIFORMLY PERSISTENT SEMIDYNAMICAL SYSTEMS
}

\author{
ALESSANDRO FONDA
}

(Communicated by Kenneth R. Meyer)

\begin{abstract}
A necessary and sufficient condition is given for a semidynamical system to be uniformly persistent. As a consequence some known results are improved.
\end{abstract}

1. Introduction. The concept of persistence plays an important role in mathematical ecology. When a system of interacting species is "persistent" in a suitable strong sense, it is quite sure that no species will go to an extinction in the future. Various definitions of persistence have been considered in the literature in order to study the behavior of the solutions of differential systems modeling biological or chemical situations (see e.g. $[\mathbf{3}, \mathbf{5}]$ and the references therein). Among these, that of uniform persistence seems to be the most suitable from the point of view of applications, since it describes a situation more likely to be maintained under small perturbations due to stochastic effects. A semiflow on a closed subset $E$ of a locally compact metric space is said to be uniformly persistent if the boundary of $E$ is "repelling" in a suitable strong sense (see $[\mathbf{3}, \mathbf{4}]$ ).

The concept of uniform persistence was first introduced in [18] in order to study the behavior of hypercycles. Different terms were used since then: "cooperativeness" (e.g. in $[\mathbf{9}, \mathbf{1 0}]$ ), "permanent coexistence" (e.g. in [14]), "permanence" (e.g. in $[\mathbf{1 1}, \mathbf{1 6}])$. A general theorem presented by Hofbauer in [9], and later improved by Hutson [12], has found many applications to population dynamics (see e.g. [1, 14, 19]).

Recently Butler, Freedman and Waltman [3] proved that, under certain conditions, uniform persistence is equivalent to weaker forms of persistence. With essentially the same assumptions, Butler and Waltman [4] were then able to obtain a necessary and sufficient condition for a dynamical system to be uniformly persistent, giving an application to a model describing the mutual interaction among three species. In [3 and 4] a fundamental hypothesis is the "acyclicity" of the flow restricted to the boundary of the considered set. There are however situations that arise rather naturally in some population models in which such a condition is not satisfied, and nevertheless uniform persistence still occurs (see e.g. Kirlinger [16], where a four-species differential system is analyzed).

In this paper we prove a theorem which provides a necessary and sufficient condition for a semidynamical system to be uniformly persistent. More generally, we give a characterization of uniform repulsivity for a compact set. The result is rather different from the one in [4], since only "local" conditions are required. The proof uses only elementary topological arguments. Nevertheless the result improves

Received by the editors May 15, 1986 and, in revised form, June 16, 1987.

1980 Mathematics Subject Classification (1985 Revision). Primary 34C35, 58F35; Secondary $92 \mathrm{~A} 15$. 
the previous theorems of Hofbauer and Hutson, as is shown in Corollary 2. It is also worth mentioning that the proof of our theorem holds true even in the case of discrete semidynamical systems.

2. The main result. In this paper we consider a semidynamical system defined on a locally compact metric space $(X, d)$. By this we mean a mapping

$$
\pi: X \times \mathbf{R}^{+} \rightarrow X, \quad \pi(x, t)=x t
$$

which is continuous and such that $x 0=x$ and $x\left(t_{1}+t_{2}\right)=\left(x t_{1}\right) t_{2}$ for any $x$ in $X$ and $t_{1}, t_{2}$ in $\mathbf{R}^{+}$, the set of nonnegative real numbers. The positive orbit of a point $x \in X$ is the set $\gamma^{+}(x)=\{x t: t \geq 0\}$. A set $E \subset X$ is positively invariant if $\gamma^{+}(x)$ is contained in $E$ for each $x \in E$.

DEFINITION 1. A subset $S$ of $X$ is said to be a uniform repeller iff there exists an $\eta>0$ such that for all $x \in X \backslash S, \liminf _{t \rightarrow+\infty} d(x t, S) \geq \eta$.

THEOREM 1. Let $S$ be a compact subset of $X$ such that $X \backslash S$ is positively invariant. A necessary and sufficient condition for $S$ to be a uniform repeller is that there exist a neighborhood $U$ of $S$ and a continuous function $P: X \rightarrow \mathbf{R}^{+}$satisfying the following conditions:

(a) $P(x)=0 \Leftrightarrow x \in S$,

(b) $(\forall x \in U \backslash S)\left(\exists T_{x}>0\right): P\left(x T_{x}\right)>P(x)$.

ProOF. To prove the necessity, assume $S$ to be a uniform repeller. Let $\eta>0$ be like in Definition 1. Define $P(x)=d(x, S)$ and $U=\{x \in X: P(x)<\eta / 2\}$. Then $P$ is continuous, (a) holds and $U$ is a neighborhood of $S$. Moreover, taken $x \in U \backslash S$, as a consequence of Definition 1 there exists a $t_{x} \geq 0$ such that

$$
P\left(x t_{x}\right)=d\left(x t_{x}, S\right)>\eta / 2>P(x) .
$$

So (b) holds with $T_{x}=t_{x}$, and the necessity is proved.

To prove the sufficiency, we have to consider the following sets

$$
I(p)=\{x \in X: P(x) \leq p\}
$$

where $p$ is a positive real number. The continuity of $P$ and (a) imply that $I(p)$ is a closed neighborhood of $S$. Moreover, since $S$ is a compact set in a locally compact space, $I(p)$ is compact when $p>0$ is sufficiently small. Let us fix $p>0$ such that $I(p)$ is a compact set contained in $U$. For $q \in] 0, p[$, we set $V(q)=\{x \in X: q \leq$ $P(x) \leq p\}$. We need the following

Claim. For every $q \in] 0, p[$ there exists a $T>0$ such that, taken any $x \in V(q)$ there is a $t_{x} \in[0, T]$ such that $x t_{x} \notin I(p)$.

Let us prove the Claim. Fix $q \in] 0, p[$. We are going to construct an open covering of the compact set $V(q)$. By assumption (b), for any $y \in I(p) \backslash S$ one can find a $T_{y}>0$ and a real number $h_{y}>1$ such that $P\left(y T_{y}\right)>h_{y} P(y)$. By the continuity of $P$ there is an $\varepsilon_{y}>0$ such that, for all $z \in B\left(y, \varepsilon_{y}\right)$,

$$
P\left(z T_{y}\right) \geq h_{y} P(z) \text {. }
$$

Since $V(q)$ is contained in $I(p) \backslash S$, the open balls $B\left(y, \varepsilon_{y}\right)$, as $y$ varies in $I(p) \backslash S$, cover $V(q)$. There exists then a finite subcovering $\left\{B\left(y_{i}, \varepsilon_{y i}\right): i=1, \ldots, m\right\}$ of $V(q)$. Defining $h=\min \left\{h_{y i}: i=1, \ldots, m\right\}$, we have $h>1$ and, by (1),

$$
(\forall z \in V(q))(\exists i \in\{1, \ldots, m\}): P\left(z T_{y i}\right) \geq h P(z) .
$$


As a consequence, setting $T_{M}=\max \left\{T_{y i}: i=1, \ldots, m\right\}$, we have

$$
(\forall z \in V(q))\left(\exists \mathscr{T} \in\left[0, T_{M}\right]\right): P(z \mathscr{T}) \geq h P(z) .
$$

Let now $n \in \mathbf{N}$ be such that

$$
h^{n-1} q \leq p<h^{n} q
$$

and set $T=n T_{M}$. We will check that $T$ verifies the Claim. Suppose by contradiction that there exists $x \in V(q)$ such that $x t \in I(p)$ for all $t \in[0, T]$. Since $x \in V(q)$, by (2) we have

$$
\exists \mathscr{T}_{1} \in\left[0, T_{M}\right]: P\left(x \mathscr{T}_{1}\right) \geq h P(x) \geq h q .
$$

If $n=1$, then by (3) $P\left(x \mathscr{T}_{1}\right)>p$, i.e. $x \mathscr{T}_{1} \notin I(p)$ and we get the contradiction. If $n>1$, since we are supposing $x t \in I(p)$ for all $t \in[0, T]$ and $\mathscr{T}_{1} \leq T_{M} \leq T$, we have $x \mathscr{T}_{1} \in V(q)$. Hence by (2)

$$
\exists \mathscr{T}_{2} \in[0, T]: P\left(\left(x \mathscr{T}_{1}\right) \mathscr{T}_{2}\right) \geq h P\left(x \mathscr{T}_{1}\right) \geq h^{2} q .
$$

If $n=2$, then by $(3) P\left(x\left(\mathscr{T}_{1}+\mathscr{T}_{2}\right)\right)>p$, i.e. $x\left(\mathscr{T}_{1}+\mathscr{T}_{2}\right) \notin I(p)$, and this is a contradiction since $\mathscr{T}_{1}+\mathscr{T}_{2} \leq 2 T_{M}=T$. If $n>2$, we repeat the same argument. At last we get

$$
\exists \mathscr{T}_{n} \in[0, T]: P\left(x\left(\mathscr{T}_{1}+\cdots+\mathscr{T}_{n}\right)\right) \geq h^{n} q>p
$$

and $\mathscr{T}_{1}+\cdots+\mathscr{T}_{n} \leq n T_{M}=T$. Hence $x\left(\mathscr{T}_{1}+\cdots+\mathscr{T}_{n}\right) \notin I(p)$, a contradiction. The Claim is thus proved.

We are now able to conclude the proof of Theorem 1. First of all we notice that for every $x \in I(p) \backslash S$ we can find a $t_{x} \geq 0$ such that $x t_{x} \notin I(p)$. It is sufficient to apply the Claim by taking $q=P(x)$ to see this. Next we consider the points outside $I(p)$, and we notice that either their positive orbit stays always outside $I(p)$, or it enters $V(\bar{q})$ for a certain fixed $\bar{q} \in] 0, p[$ (e.g. $\bar{q}=p / 2$ ). The claim then gives us a $T>0$ such that, whenever an orbit enters in $V(\bar{q})$, it must go out of $I(p)$ after a time at most equal to $T$ ( $T$ is independent of the points in $V(\bar{q}))$. The set

$$
\Gamma=\{x t: x \in V(\bar{q}), t \in[0, T]\}
$$

is compact and has empty intersection with $S$. Hence choosing

$$
\eta=\min \{d(s, \gamma): s \in S, \gamma \in \Gamma\},
$$

by what has been said above, $\eta$ is positive and satisfies Definition 1 .

REMARK 1. If $E$ is a positively invariant closed subset of $X$, one can restrict the study of the semidynamical system to $E$. The system on $E$ is said to be uniformly persistent if and only if the boundary of $E$ is a uniform repeller in the sense of Definition 1. Here and in the following corollaries we give conditions for a compact set $S$ to be a uniform repeller. It is straightforward, though, to obtain the analogous conditions for the system to be uniformly persistent on a closed positively invariant set $E$ with compact boundary.

REMARK 2. When $S$ is a compact set, assumption (b) is equivalent to the following one, which perhaps is easier to verify in applications.

$\left(\mathrm{b}^{\prime}\right)(\forall x \in S)\left(\exists \varepsilon_{x}>0\right)\left(\forall y \in B\left(x, \varepsilon_{x}\right) \backslash S\right)(\exists T>0): P(y T)>P(y)$.

REMARK 3. It is easy to see that Theorem 1 holds true also for discrete semidynamical systems $\left\{F^{k}: k=0,1, \ldots\right\}$, where $F: X \rightarrow X$ is a continuous map, $X$ 
is a compact set and (b) is replaced by

$\left(\mathrm{b}^{\prime \prime}\right)(\forall x \in U \backslash S)(\exists k \in\{1,2, \ldots\}): P\left(F^{k} x\right)>P(x)$.

The proof is a direct modification of the one given above.

REMARK 4 . Theorem 1 can be easily extended to local semidynamical systems (cf. [2]), provided that Definition 1 is adapted in the obvious way. Indeed, if $S$ is compact, since $X$ is locally compact, there is $\eta>0$ such that $\{y \in X: d(y, S) \leq \eta\}$ is compact. Hence $\liminf _{t \rightarrow \omega_{x}} d(x t, S)>\eta$ for each $x \in X \backslash S$ such that $\omega_{x}<+\infty$. Then it is sufficient to analyze the orbits of those $x$ for which $\omega_{x}=+\infty$.

REMARK 5. In the proof of Theorem 1 we never consider the orbit of any point of the set $S$ : the result still holds if the semidynamical system is defined only on $X \backslash S$. Hence Theorem 1 is independent of the result in [4], where an acyclic condition is required for the flow on $S$. We further remark that the hypothesis in [4] of dissipativeness of the flow is here substituted by the compactness of the set $S$. As far as applications are concerned, however, one usually can assume the system to be defined on a compact set $E$, in which case they are both satisfied taking $S$ to be the boundary of $E$ (see Remark 1).

We now give a simple consequence of Theorem 1 . In what follows, $S$ will always denote a compact subset of $X$ such that $X \backslash S$ is positively invariant.

COROLlaRY 1. Let $S$ be as above. If for every $x \in S$ there is a $T_{x}>0$ such that $x T_{x} \in X \backslash S$, then $S$ is a uniform repeller.

ProOF. We will apply Theorem 1 by choosing $P(x)=d(x, S)$. By Remark 2 it is then sufficient to verify condition $\left(\mathrm{b}^{\prime}\right)$. Fix $x \in S$; by the hypothesis, one can choose $T_{x}>0$ and $\eta_{x}>0$ such that $d\left(x T_{x}, S\right)>2 \eta_{x}$. By continuity, there exists $\varepsilon_{x} \leq \eta_{x}$ such that $y T_{x} \in B\left(x T_{x}, \eta_{x}\right)$ whenever $y \in B\left(x, \varepsilon_{x}\right)$. Setting $T=T_{x}$,

$$
P(y T)=d(y T, S)>d(x T, S)-\eta_{x}>\eta_{x}>d(y, S)=P(y) .
$$

So $\left(b^{\prime}\right)$ is verified and the proof is complete.

We now give a generalization of a result of Hutson [12, Theorem 2.5] which includes a previous theorem of Hofbauer [9]. By $\Omega(S)$ we denote the whole $\omega$-limit of the orbits on $S$, i.e. $\Omega(S)=\left\{z \in X: \exists x \in S,\left(t_{n}\right)_{n}, t_{n} \rightarrow+\infty, z=\lim _{n \rightarrow \infty} x t_{n}\right\}$, and we denote by $\bar{\Omega}(S)$ its closure.

Since we have to consider differentiation along the orbits, we will suppose for simplicity $X$ to be a (closed) positively invariant subset of $\mathbf{R}^{n}$, and the system considered to be the dynamical system generated by an autonomous ODE in $\mathbf{R}^{n}$. We denote differentiation along the orbits by a dot "." (see [17, p. 29]).

COROllary 2. Let $S$ be as above, and $P \in C\left(X, \mathbf{R}^{+}\right) \cap C^{1}(X \backslash S, \mathbf{R})$ be such that $P(x)=0 \Leftrightarrow x \in S$. Moreover, let there exist a lower semicontinuous function $\psi: X \rightarrow \mathbf{R}$, bounded below, and an $\alpha \in[0,1]$ such that

(i) $\dot{P}(x) \geq[P(x)]^{\alpha} \psi(x) \forall x \in X \backslash S$,

(ii) $\forall x \in \Sigma, \sup _{T \geq 0} \int_{0}^{T} \psi(x s) d s>0$,

where $\Sigma$ denotes $S$ or, whenever $S$ is positively invariant, $\bar{\Omega}(S)$. Then $S$ is a uniform repeller.

PROOF. Let us first consider the case $\Sigma=S$. In order to verify condition $\left(\mathrm{b}^{\prime}\right)$, let us fix $x \in S$. By (ii), there exists $T>0$ such that $\int_{0}^{T} \psi(x s) d s>0$. By the 
lower semicontinuity of $\psi$, there exists $\varepsilon_{x}>0$ such that, for every $y \in B\left(x, \varepsilon_{x}\right) \backslash S$, we have

$$
0<\int_{0}^{T} \psi(y s) d s \leq \int_{0}^{T} \frac{\dot{P}(y s)}{[P(y s)]^{\alpha}} d s= \begin{cases}\frac{1}{1-\alpha}\left[P(y T)^{1-\alpha}-P(y)^{1-\alpha}\right] & \text { if } \alpha<1 \\ \log \frac{P(y T)}{P(y)} & \text { if } \alpha=1 .\end{cases}
$$

So $\left(\mathrm{b}^{\prime}\right)$ holds and the corollary follows from Theorem 1 .

Consider now the case $\Sigma=\bar{\Omega}(S)$. By Fatou's Lemma, the function $y \rightarrow$ $\int_{0}^{T} \psi(y s) d s$ is lower semicontinuous (see [12, Lemma 2.4]). So also the map

$$
y \rightarrow \sup _{T \geq 0} \int_{0}^{T} \psi(y s) d s
$$

is lower semicontinuous, and since $\bar{\Omega}(S)$ is compact, by (ii) there exist $\delta>0$ and an open set $W \supset \bar{\Omega}(S)$ such that

$$
\sup _{T \geq 0} \int_{0}^{T} \psi(y s) d s>\delta \quad \forall y \in W .
$$

Fix now $x \in S$. Since $W$ is open and contains $\bar{\Omega}(S)$, there exists a $t_{x} \geq 0$ such that $x t \in W$ for all $t \geq t_{x}$, so that by repeated use of (4) we obtain

$$
\sup _{T \geq 0} \int_{0}^{T} \psi(x s) d s>0 .
$$

Then (ii) holds with $\Sigma=S$ and the first part of the proof gives us the result.

REMARK 6. It is not difficult to generalize assumption (ii) in Corollary 2 , in the spirit of condition $\left(\mathrm{b}^{\prime}\right)$. In such a way the choice of a $\psi$ identically zero on $S$ is admitted provided that $\psi>0$ "near" $S$. This seems to slightly generalize the theorems of Hofbauer and Hutson.

In the following corollary we consider the case of a finite number of functions $V_{i}$ having properties similar to the function $P$ of Corollary 2 .

COROLlaRY 3. Let $S$ be as above and suppose there exist $V_{1}, \ldots, V_{k} \in C\left(X, \mathbf{R}^{+}\right)$ $\cap C^{1}(X \backslash S, \mathbf{R})$ satisfying the following properties:

(i) $V_{i}(x)>0 \forall x \in X \backslash S, \forall i$,

(ii) $(\forall x \in S)(\exists i \in\{1, \ldots, k\}): V_{i}(x)=0$.

Moreover let there exist lower semicontinuous functions $\psi_{i}: X \rightarrow \mathbf{R}$ and $\alpha_{i} \geq 0$ $(i=1, \ldots, k)$ such that

(iii) $V_{i}(x)=0 \Rightarrow \psi_{i}(x)>0$,

(iv) $\dot{V}_{i}(x) \geq\left[V_{i}(x)\right]^{\alpha_{i}} \psi_{i}(x) \forall x \in X \backslash S$.

Then $S$ is a uniform repeller.

Proof. In order to apply Theorem 1 , define $P(x)=\min \left\{V_{i}(x): i=1, \ldots, k\right\}$. Clearly $P$ is continuous and verifies (a). Let us then verify $\left(\mathrm{b}^{\prime}\right)$. Fix $x \in S$. We can assume without restrictions that, for a certain $r \in\{1, \ldots, k\}$,

$$
V_{1}(x)=\cdots=V_{r}(x)=0 ; \quad V_{i}(x) \neq 0 \forall i>r .
$$

By (i), (iii), (iv) and the lower semicontinuity of $\psi_{i}$, there exists $\eta_{x}>0$ such that $\dot{V}_{i}(y)>0$ for any $y \in B\left(x, \eta_{x}\right) \backslash S$ and $i \in\{1, \ldots, r\}$. It follows easily that there 
exists $\left.\varepsilon_{x} \in\right] 0, \eta_{x}\left[\right.$ such that for each $y \in B\left(x, \varepsilon_{x}\right) \backslash S$ there is a $T>0$ sufficiently small such that $P(y T)>P(y)$, and the proof is complete.

ACKNowledgment. The author would like to thank Professor F. Zanolin for very useful discussions on the subject of this paper.

\section{REFERENCES}

1. E. Amann and J. Hofbauer, Permanence in Lotka-Volterra and replicator equations, Proc. Conf. Wartburg, G.D.R., Akademie-Verlag, Berlin, 1985, pp. 23-34.

2. N. P. Bhatia and O. Hajek, Local semi-dynamical systems, Springer-Verlag, Berlin-HeidelbergNew York, 1969.

3. G. J. Butler, H. I. Freedman and P. Waltman, Uniformly persistent systems, Proc. Amer. Math. Soc. 96 (1986), 425-430.

4. G. J. Butler and P. Waltman, Persistence in dynamical systems, J. Differential Equations 63 (1986), 255-263.

5. M. L. Fernandes and F. Zanolin, Remarks on strongly flow-invariant sets, J. Math. Anal. Appl. (to appear).

6. __ Repelling conditions for boundary sets using Liapunov-like functions (in preparation).

7. H. I. Freedman and W. H. So, Global stability and persistence of simple food chain, Math. Biosci. 76 (1985), 69-86.

8. H. I. Freedman and P. Waltman, Persistence in models of three interacting predator-prey populations, Math. Biosci. 68 (1984), 213-231.

9. J. Hofbauer, A general cooperation theorem for hypercycles, Monatsh. Math. 91 (1981), 233240.

10. J. Hofbauer, P. Schuster and K. Sigmund, Competition and cooperation in catalitic selfreplication, J. Math. Biol. 11 (1981), 155-168.

11. J. Hofbauer and K. Sigmund, Permanence for replicator equations, Proc. Math. Dynamic Processes, Lecture Notes in Math., Springer-Verlag (to appear).

12. V. Hutson, A theorem on average Liapunov functions, Monatsh. Math. 98 (1984), 267-273.

13. V. Hutson and W. Moran, Persistence of species obeying difference equations, J. Math. Biol. 15 (1982), 203-231.

14. V. Hutson and G. T. Vickers, A criterion for permanent coexistence of species, with application to a two-prey one-predator system, Math. Biosci. 63 (1983), 253-269.

15. V. Hutson and R. Law, Permanent coexistence in general models of three interacting species, J. Math. Biol. 21 (1985), 285-298.

16. G. Kirlinger, Permanence in Lotka-Volterra equations: linked prey-predator systems, Math. Biosci. 81 (1986), 1-27.

17. J. P. La Salle, The stability of dynamical systems, CBMS Regional Conf. Ser. in Appl. Math., SIAM, Philadelphia, 1976.

18. P. Schuster, K. Sigmund and R. Wolff, Dynamical systems under constant organization. III: Cooperative and competitive behavior of hypercycles, J. Differential Equations 32 (1979), 357368.

19. K. Sigmund and P. Schuster, Permanence and uninvadability for deterministic population models, Springer Series in Synergetics, Vol. 21, Springer-Verlag, Berlin and New York, 1984, pp. 173184.

DEPARTMENT OF MATHEMATICS, S.I.S.S.A., INTERNATIONAL SCHOOL FOR ADVANCED Studies, Strada Costiera 11, 34014 Trieste, ItAly 